\title{
Measurement of Surge Propagation in Induction Machines
}

\author{
T. Humiston, Student Member, IEEE \\ Department of Electrical and Computer Engineering \\ Clarkson University \\ Potsdam, NY 13699
}

\author{
P. Pillay, Senior Member, IEEE \\ Department of Electrical and Computer Engineering \\ Clarkson University \\ Potsdam, NY 13699
}

\begin{abstract}
Winding failures in induction machines have been a major concern in the past several years, and more so recently with the addition of variable speed drives (VSDs). Both the introduction of the vacuum breaker, and the use of pulse width modulation (PWM) drives, utilizing fast switching IGBT's, have resulted in an increase in winding failures in induction machines.
\end{abstract}

Two mechanisms that cause winding failures are steepfronted surges, like those caused during the opening and closing of vacuum breakers, and transient overvoltages caused by impedance mismatch between the cable and load during VSD operation. There has been a fair amount of work done to date on the propagation of vacuum breaker induced steep-fronted surges in the windings of the induction machine. More recently, work has been progressing on overvoltages at the machine terminals as a result of VSD's operating with long cables connecting drive and machine. However the propagation of these surges down the coils and the interference of these PWM surges with each other in the coils as well as the coupling between turns and coils have not been thoroughly investigated. Such an investigation would allow more benign PWM strategies to be developed, which do not build up in the machine to unacceptably high levels. To this end, this paper presents a measurement technique which can be used to study PWM generated surges and their propagation within the coil, considering each turn at a time. This requires the inclusion of the differences in the surge impedances in each section of the coil.

Detailed parameter measurements are made of each turn section (slot vs. overhang), which are then used to determine the surge impedance of each section. Reflection and refraction coefficients are calculated, then used to map, via lattice diagrams, the propagation of surges within the turns of one coil.

\section{INTRODUCTION}

Winding failures in induction machines have been a major concern in the past several years, and more so recently with the addition of variable speed drives (VSDs). Both the introduction of the vacuum breaker, and the introduction of PWM drives, utilizing fast switching IGBT's, have resulted in an increase in winding failures in induction machines.

There are two mechanisms causing winding failures; steep-fronted surges, like those caused during the opening and closing of vacuum breakers, and transient overvoltages caused by impedance mismatch between cable and load during VSD operation. There has been a fair amount of work done to date on the propagation of vacuum breaker induced steep-fronted surges in the windings of large induction machines[5,6,8-12]. The majority of this work has focused on a single event surge entering one winding of a multiphase machine. In addition, the winding parameters have been limited to calculated values. Here the intention is to study the propagation of multiple surges in the machine, their interaction through mutual inductive and capacitance coupling and when operated from individual single phase inverters supplying each phase.

The machine under study is form-wound, hence parallel plate capacitance theory has been applied as shown in figure 1 .
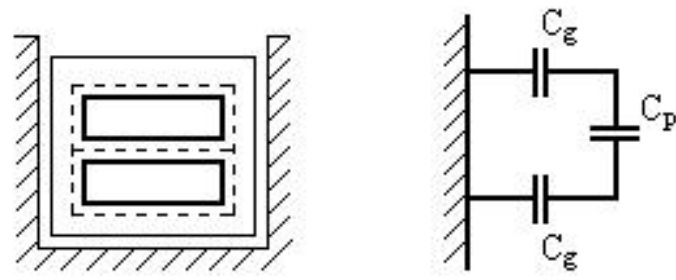

Figure 1. Capacitance in a 2-turn coil.

The individual values of $\mathrm{C}_{\mathrm{g}}$ and $\mathrm{C}_{\mathrm{p}}$ are calculated using parallel plate approximations and the $\epsilon_{\mathrm{r}}$ value of the respective insulations. This can give reasonably accurate capacitance values. But as for inductance values, some have used finite element methods[8], but most have used the equation

$$
[\mathrm{L}]=\left[\mathrm{C}_{0}\right]^{-1} / \mathrm{c}^{2}
$$

where $[\mathrm{L}]$ is the inductance matrix, $\left[\mathrm{C}_{0}\right]$ is the capacitance matrix found as mentioned above with the exception that all $\epsilon_{\mathrm{r}}=1$, and $\mathrm{c}$ is the velocity of propagation [6]. This method requires knowledge of the velocity of propagation in the coils. One study [6], assumed the wave propagation velocity in the overhang section of the coil is the same as within the slot region. This assumption is not supported by [9] which report the velocity of propagation in the slot as been lower than in the overhang.

More recently, work has been progressing on overvoltages at the machine terminals as a result of VSD's operating with long cables connecting drive and machine [14]. The long cables have an impedance significantly different 
from the impedance of the induction machine. When a steep fronted wave traveling along the cable encounters the impedance mismatch a voltage reflection occurs resulting in an overvoltage at the location of the mismatch, i.e. the machine terminals. As switching frequencies increase so do the rise times on the devices, translating into a higher $\mathrm{dv} / \mathrm{dt}$ pulse/wave traveling down the cable. The higher the $\mathrm{dv} / \mathrm{dt}$ the steeper the wave front impacting the impedance mismatch. Thus, peak overvoltages increase as the rise time of semiconductor devices reduces [1]. The result is that voltage reflections are dependent on VSD output pulse rise time and cable length.

The work further focuses on the measurement of surge propagation, differentiating between the impedance of the slot sections and that of the overhang sections, of each turn. Detailed parameter measurements are made of each turn section (slot vs. overhang), which are then used to determine the surge impedance of each section. Reflection and refraction coefficients are calculated, then used to map, via lattice diagrams, the propagation of surges within the turns of one coil.

The paper is divided into six sections. The first is on parameter measurements and the method used to obtain the measurements. Second, experimental set up for surge measurements. Third, single section lattice diagram simulation and measurement. Fourth, the Bewely lattice diagram is extended to map all sections of a coil within the induction machine. Fifth, PWM pulse tracking within the turns of a coil using a lattice diagram. And Sixth, the conclusions.

\section{PARAMETER MEASUREMENTS}

The stator coils sit in slots, where the coils are surrounded on three sides by iron; in the overhang the coils are surrounded by air only. These two distinctly different environments cause the coils to have different surge impedances. For the slot region, because of the presence of the iron the inductance is much higher than for the overhang region. The effect on the capacitance is also greater in the slot, than in the overhang where the only coil coupling is to the adjacent coil. These regions become important when steep-fronted surges attempt to pass between them. An incident surge will experience reflections, refractions and cause overvoltages at impedance mismatches.

There are also mismatches at the phase terminals, where the connection from the terminal meets the coil. The coil can be divided into six distinct regions of differing impedances. Since the wave front duration is much shorter than the coil propagation time each turn acts as a separate conductor, even though they are connected to one another. However, this only holds during the transient period of a few microseconds. Because of this, a coil can be modeled like a multiconductor transmission line [8-10] with many series segments consisting of slot sections and overhang regions. The theories of wave propagation on multiconductor transmission lines can be applied to the coils of a machine.
Figure 2 shows a diagram of a three-turn coil as segmented into the six regions, with slot and overhang regions marked.
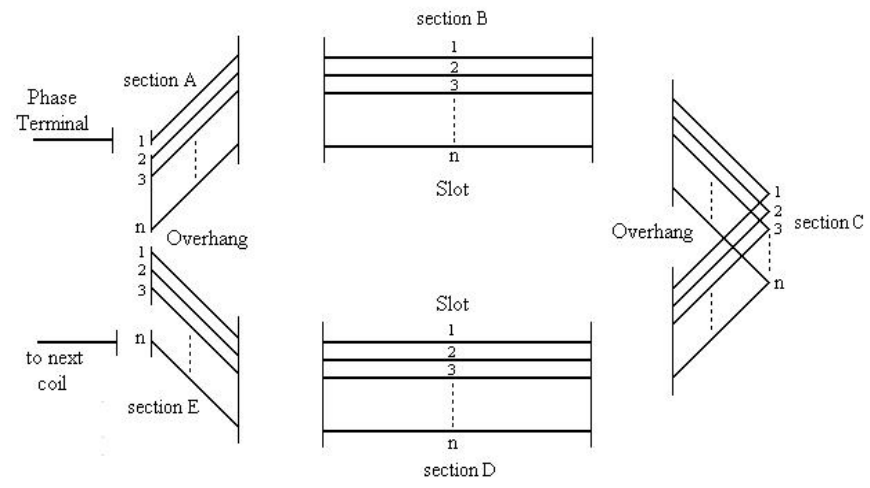

Figure 2. Multiconductor transmission model of induction motor coil with series sections.

In previous work done, the capacitance and inductance values for the different regions were calculated. In this work the coil parameters are measured. A $150 \mathrm{hp}, 3$-phase, form wound induction machine was obtained for use as the test motor. To perform the required measurements the machine was disassembled down to the stator and frame, with only the coils left in place, as shown in figure 3 . It has been shown in previous work $[5,6,8,9,11]$ that it is not necessary to have the rotor present when performing experimental research on surges within the stator windings.

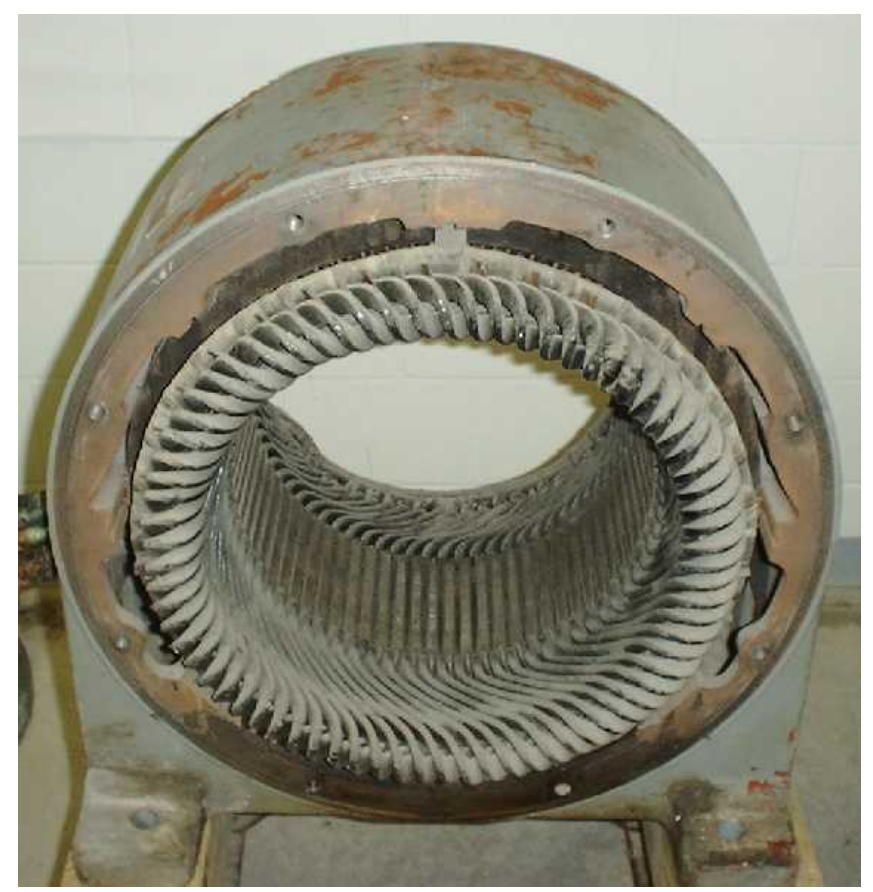

Figure 3. $150 \mathrm{HP}, 3$-phase, form wound induction motor used as test motor.

In addition because of the air gap the effect of the rotor on the parameter measurements is negligible. Several coil sections were removed to gain clear access to the stator core from each end for measurement purposes. Figure 4 shows one complete coil as removed from the machine. 


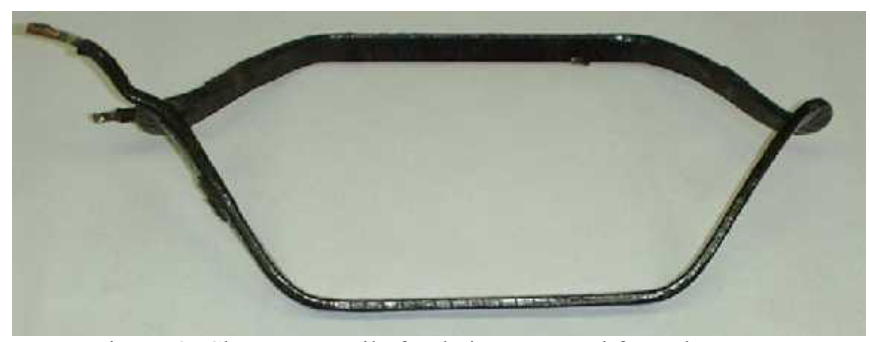

Figure 4. Shows one coil after being removed from the stator.

Six regions in each coil are identified as having a different impedance from one region to the next. However, this does not necessarily imply that there are six different sets of parameters to measure. The two slot sections (B and D figure 2) of each coil will be considered the same, as they are the same size and composed of the same materials. Likewise, overhang sections $\mathrm{A}$ and $\mathrm{E}$, figure 2, are the same with the exception of their incoming and outgoing connections. To obtain each section's parameters the coil must be cut into the respective regions and each placed back into its working environment. Figure 5 shows the actual test machine coil cut into the sections representing the different impedance values as shown in figure 2 .

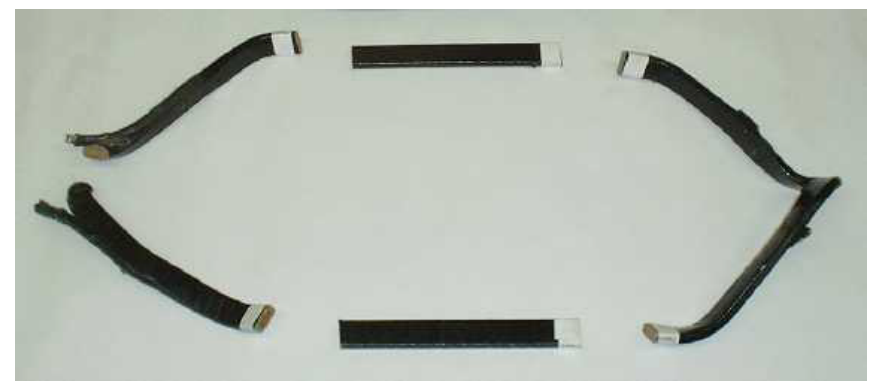

Figure 5. Test coil cut into sections representing different zones of impedance.

The parameter values were obtained using an Hewlett Packard 4284A 20Hz-1MHz Precision LCR Meter. Due to the size of the machine the standard test leads were not used. Even though a non-standard set of test leads were used the LCR meter had a correction capability via open circuit and short circuit tests to remove any errors introduced. A test setup was built to perform the capacitance measurements in the slot sections, which were placed back into the stator core. Figure 6 shows the capacitive setup. The capacitive measurements required only two contact points between the LCR meter test leads and the device under test (DUT). For the mutual, turn to turn inductances the test set up was modified to allow for four contact points to obtain the measurements. Also each contact point had to be independently movable to enable the complete set of turns to be measured. The test setup is shown in figure 7, while an inductance measurement is shown in figure 8 .

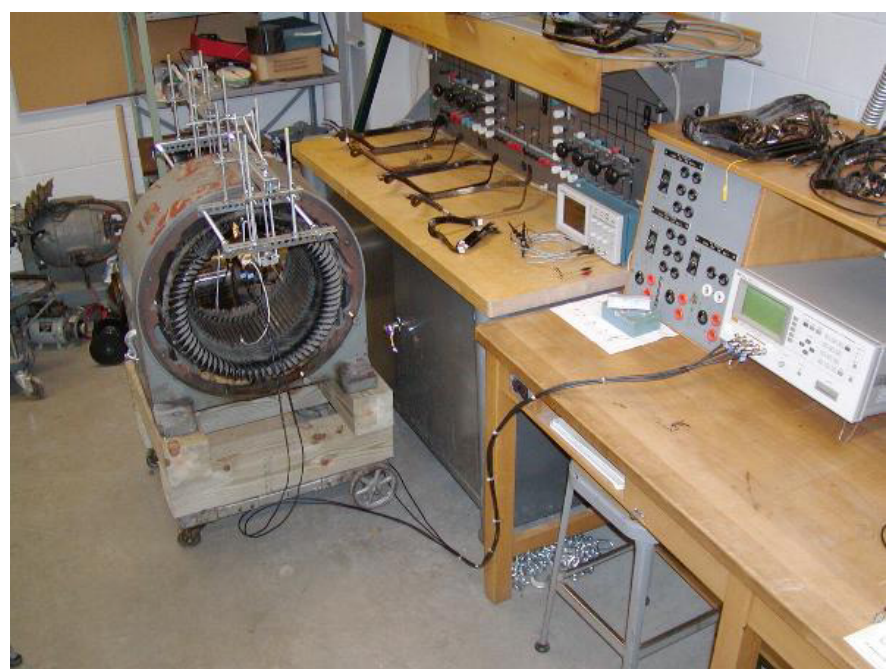

Figure 6. Test setup to measure capacitance in slot sections.

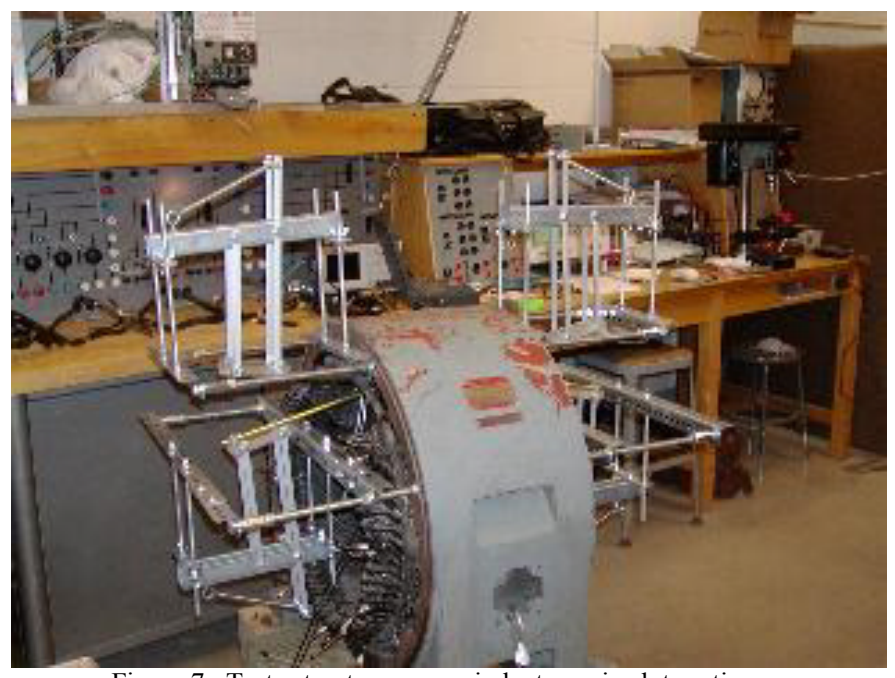

Figure 7. Test setup to measure inductance in slot sections.

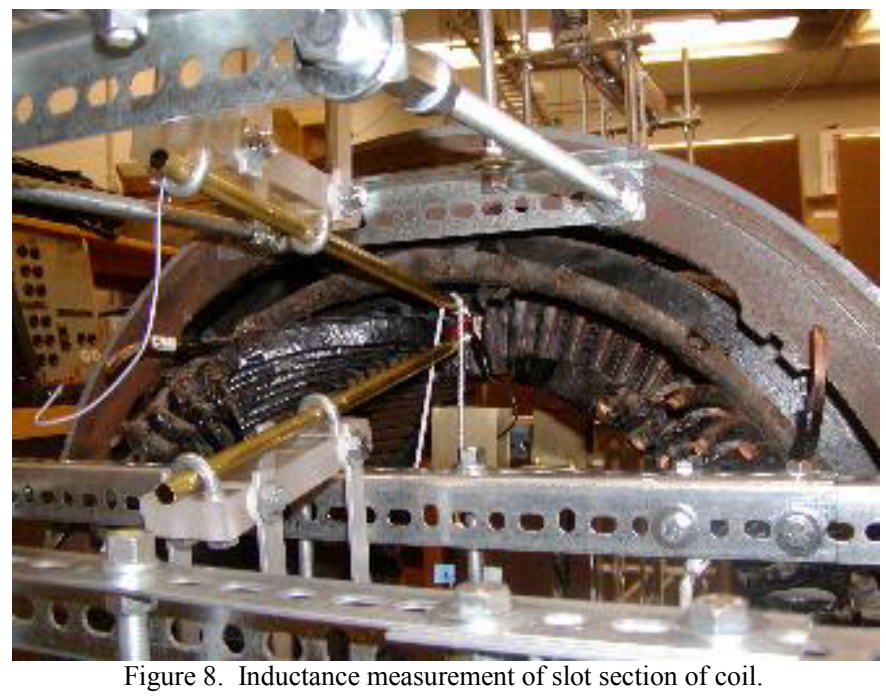

With these two setups, all turn to ground capacitances, turn to turn capacitances, self inductances and mutual inductances were measured for each conductor/turn in both the lower and 
upper coils in the double layer winding. As frequency plays an important role in impedance values all parameter measurements where taken over a range of frequencies from $60 \mathrm{~Hz}$ to $1 \mathrm{MHz}$ in ten steps, as limited by the LCR meter. The ten frequencies used were $60 \mathrm{~Hz}, 120 \mathrm{~Hz}, 500 \mathrm{~Hz}, 1 \mathrm{KHz}$, $5 \mathrm{KHz}, 10 \mathrm{KHz}, 50 \mathrm{KHz}, 100 \mathrm{KHz}, 500 \mathrm{KHz}$ and $1 \mathrm{MHz}$.

The process was repeated for the overhang sections. A jig was constructed, matching the inside shape of the stator core on to which the overhang sections were mounted. From detailed, full scale drawings, templates were made to hold the coil sections in their proper alignment. The complete jig with coil sections installed is shown in figure 9. Adjacent coils need to be in placed to account for mutual coupling as well as surge propagation from coil to coil and the impedance mismatch between coils. The adjacent phase groups must also remain in place for phase to phase coupling, as the problem occurs during normal operation. Previous research has only examined one phase.

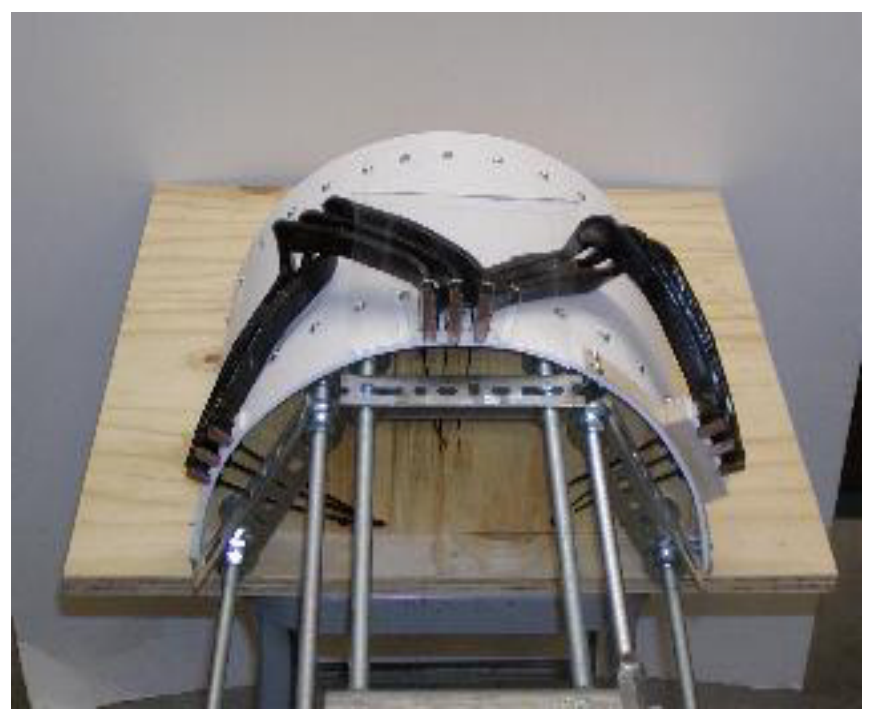

Figure 9. Form to hold overhang sections in proper alignment.

As with the slot setup, an additional support structure was needed to hold the test leads in place on the conductors while the measurements were taken. This additional structure needed to be completely and independently adjustable for each of the four contact points from test lead to the conductor being measured. Figure 10 shows the complete test setup to perform capacitance and inductance measurements on the overhang sections of the coils. Figure 11 shows a mutual inductance measurement being conducted on one of the overhang coil sections. In the slot measurements, only turn to turn and turn to ground couplings associated with the two coils residing in the slot were considered as the stator core acts as a barrier to coupling with coils in adjacent slots. Since the machine used in the experimental tests and measurements is double layer, both upper and lower coils have to be considered. Within the slot region, since both coils are parallel, a significant coupling exists between the two coils. However, in the overhang, due to the nature of the double layer winding, the upper and lower coils do not run parallel they run in opposite directions. There is only minimal overlap at the exit of the slot before turning in opposite directions. This would allow minimal coupling between upper and lower coils, and is supported by the measurements. The measured values show the coupling at a significantly low value as to be neglected.

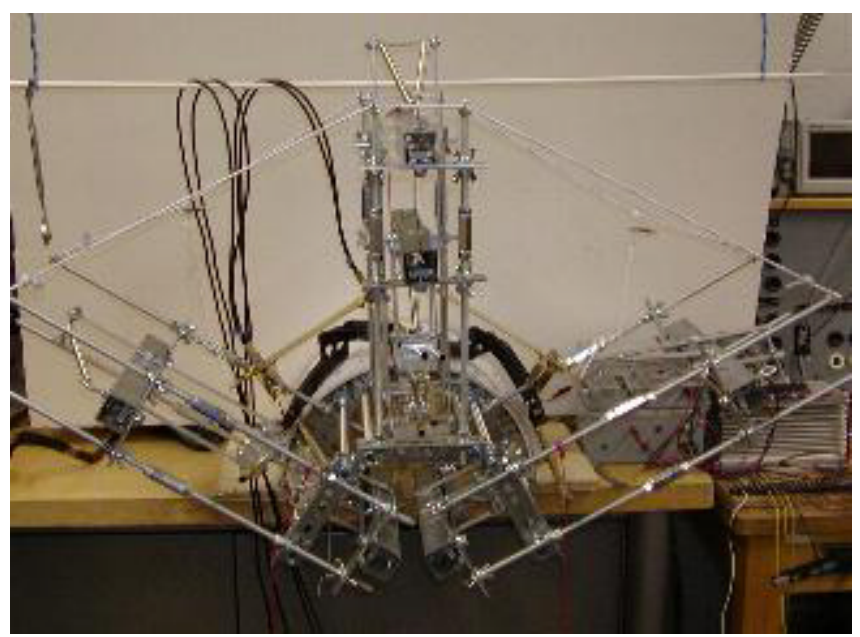

Figure 10. Test setup to measure capacitance and inductance in overhang.

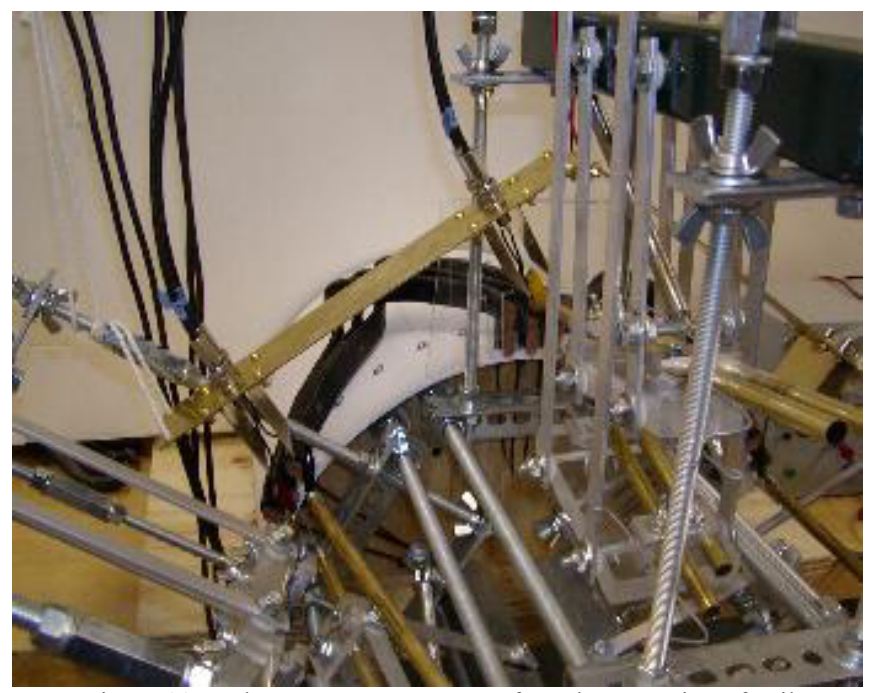

Figure 11. Inductance measurement of overhang section of coil.

It is necessary, for determining the characteristic impedance of the turn sections, to have the parameter values in units per meter. Hence, all measured data was divided by its respective conductor length, $0.3842 \mathrm{~m}$ for the overhang region and $0.1619 \mathrm{~m}$ for the slot. Figure 12 shows the self inductance of all 16 conductors ( 8 per coil) of the double layer winding for both the overhang and the slot regions in frequencies from $500 \mathrm{~Hz}$ to $1 \mathrm{Mhz}$. The left half of the figure shows the overhang values 1 to 16 (left to right), while the right half show the slot region, 1 to 16 (left to right). Figure 13 shows a comparison of the self inductance in the overhang turns versus that in the slot for a frequency of $500 \mathrm{~Hz}$ (same data as used in figure 12). The slot values show a linear decrease from turn one in the bottom of the slot to turn 16 at 
the top (nearest to rotor), as expected, while the overhang shows a reasonably constant value. Figure 14 shows that as frequency increases the inductances within the slot regions converge.

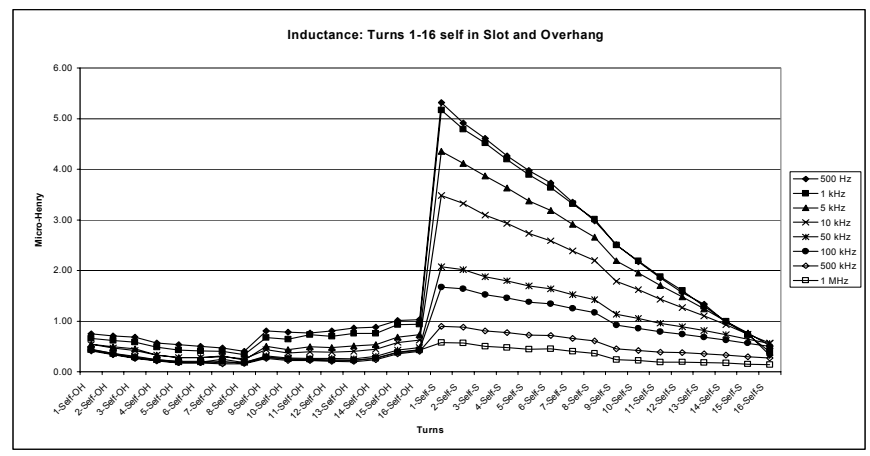

Figure 12. Measured self inductance of turns in overhang sections (left half) and in slot sections (right half).

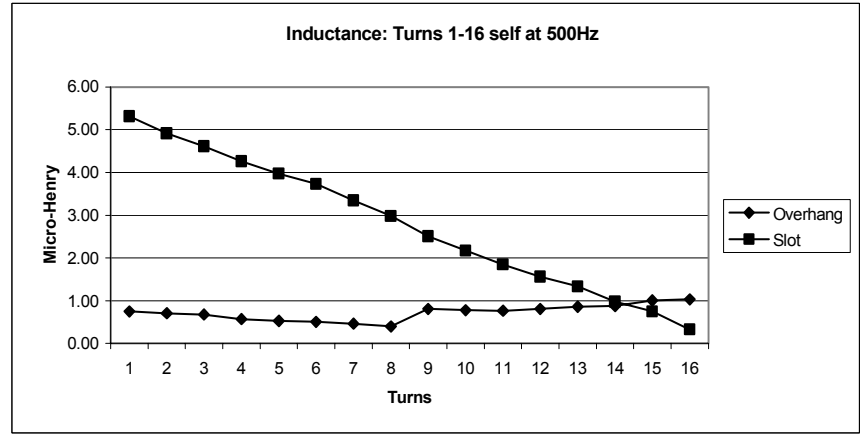

Figure 13. Comparison of self inductance in overhang vs. slot at $500 \mathrm{~Hz}$.

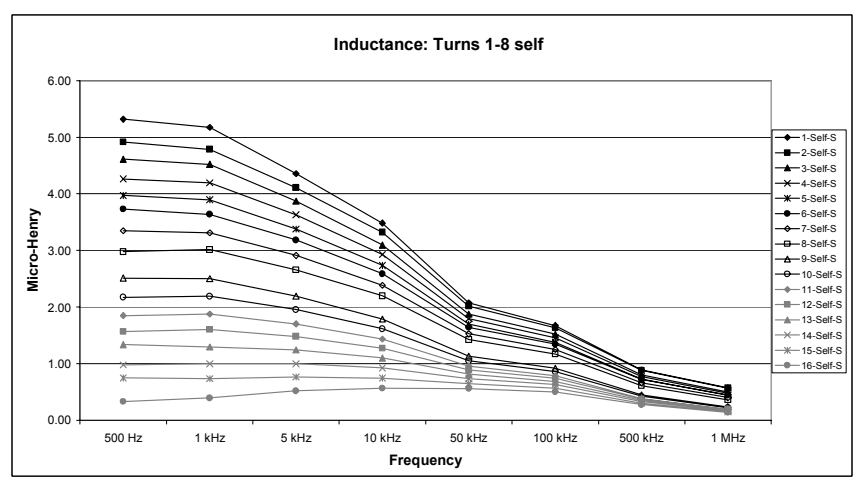

Figure 14. Self inductance in slot converging as frequency increases.

Figure 15 shows turn to adjacent turn capacitance in both the slot sections and overhang sections, for frequencies of $60 \mathrm{~Hz}$ to $1 \mathrm{MHz}$. The left half shows the slot measurements for both the lower and upper coils. The sharp drop is associated with the last turn of the lower coil and the first turn of the upper coil, where additional insulation is present. The overhang capacitance values (right half) are significantly lower than for the slot at all frequencies. Figure 16 shows as frequency is increased the slot capacitance values decrease and trend towards a constant value at high frequency. The turn to ground capacitance in the slot region also decreases as frequency increases but at a lesser rate than for the turn to turn values, as seen in figure 17.

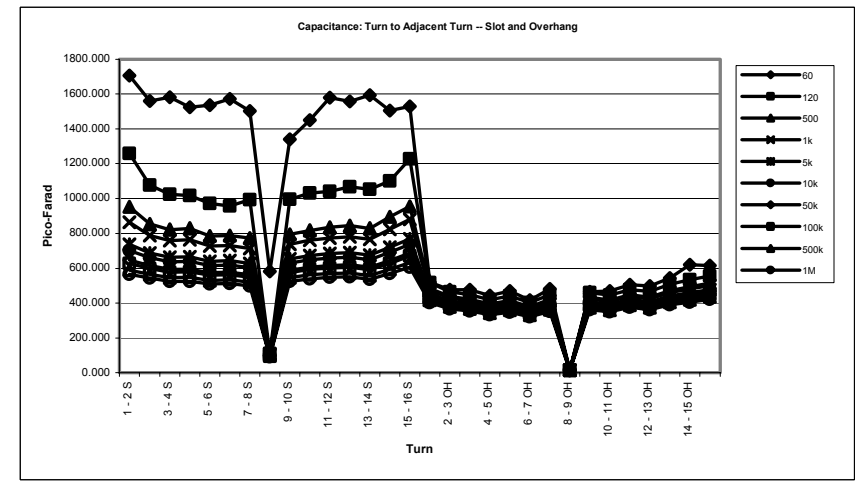

Figure 15. Measured turn to adjacent turn capacitance in slot sections (left half) and in overhang sections (right half).

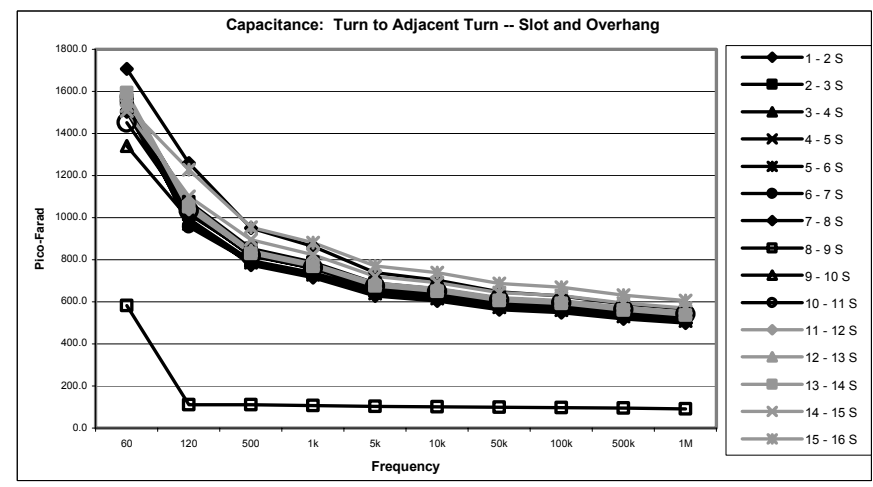

Figure 16. Turn to adjacent turn capacitance in slot converging as frequency increases.

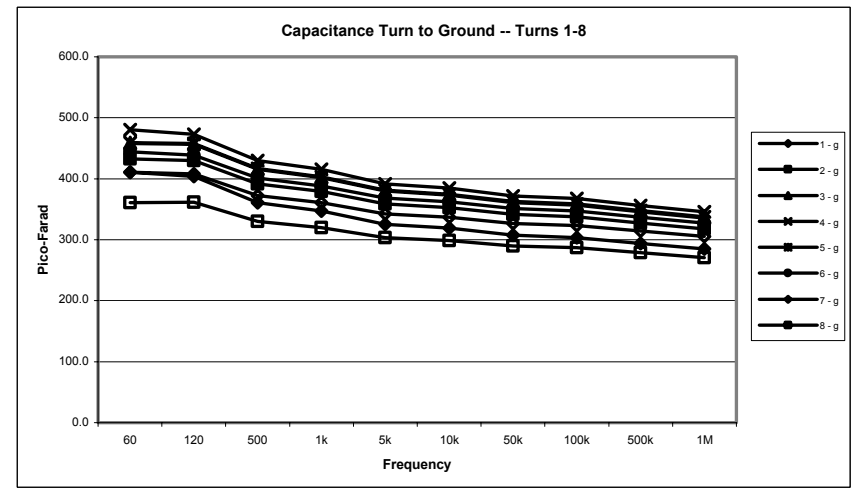

Figure 17. Turn to ground capacitance versus frequency in slot.

\section{EXPERIMENTAL SET UP FOR SURGE MEASUREMENTS}

To obtain experimental results a test set up was assembled. The two main components necessary were a source of steep fronted waves and a device to accurately measure the source and the propagating waves. For the source, two choices of function generators were used. A Tektronix FG $5033 \mathrm{MHz}$ function generator, which had a slower rise time of $54 \mathrm{nsec}$ on a pulse train waveform. The second was an Hewlett Packard 3314A function generator, which had a faster rise time of $5.2 \mathrm{nsec}$. Both generators had internal impedance values of $50 \Omega$.

For waveform measurements two CompuScope $85 \mathrm{G}$ waveform digitizers were used. Each device has two input 
channels having $5 \mathrm{GS} / \mathrm{s}$ A/D Sampling simultaneously, with $500 \mathrm{MHz}$ bandwidth and 8 bit resolution. This level of measurement capture was necessary due to the short rise times of the source, as well as the short propagation times of the coil sections. The sampled data was viewed and stored using GageScope Oscilloscope Software.

To obtain measurements of waveforms at impedance boundaries within the coil, modifications were made to allow connections from the digitizers to the coil. At the location of a boundary change, i.e. slot to overhang, the insulation was removed and small leads attached, as seen in figure 18 .

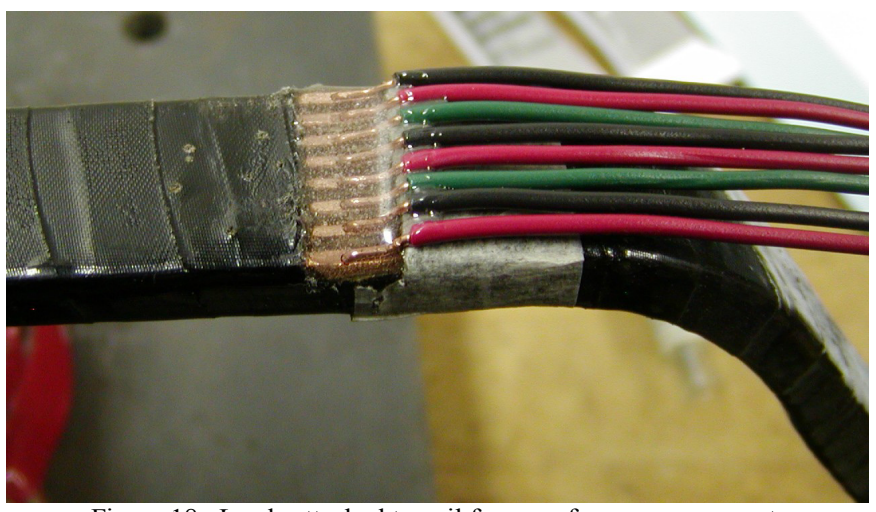

Figure 18. Leads attached to coil for waveform measurement.

\section{SINGLE SECTION LATTICE DIAGRAM}

To verify the operation of the experimental setup a simple system with conductors of known impedance values and propagation times was examined, figure 19. To the left of point ' $\mathrm{A}$ ' is the source, with internal impedance, connected to a $50 \Omega$ coaxial cable. At point 'A' the cable connects to channel 1 of the digitizer, set to an impedance of $50 \Omega$. A second, longer length, coaxial cable is

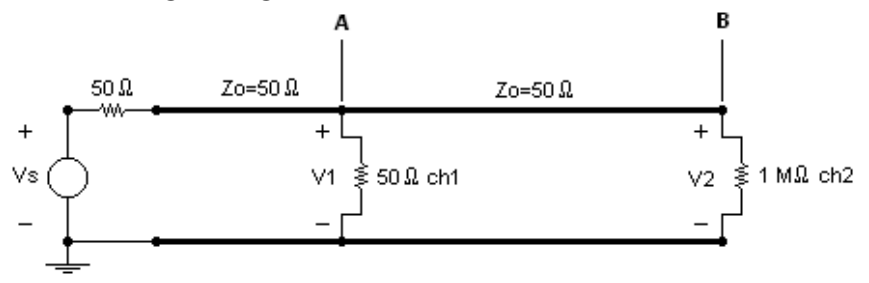

Figure 19. Simple experimental system.

connected from channel 1 to channel 2, where channel 2 is set to an impedance of $1 \mathrm{M} \Omega$, making an impedance mismatch with the cable. There is also a mismatch in impedance with point ' $A$ ' and the coaxial cable. Thus points ' $A$ ' and ' $B$ ' were used as the boundary points for a single section Bewely [13] lattice diagram analysis.

Since the rise time of the source is long compared to the propagation time of the cables, a stepped approximation of the rising edge, shown in figure 20, is used for evaluation of the wave front at the boundaries. Figure 21 shows the lattice diagram of reflections occurring between boundaries 'A' and 'B'. There are several lines, shifted in time, propagating simultaneously as a group representing the stepped edge approximation shown in figure 20. Figure 22 shows the resulting waveforms at points ' $A$ ' and ' $B$ ' for a simulated 1.5 per unit generated pulse while figure 23 shows the measured waveforms for the actual 1.5 per unit pulse. The simulation results match up accurately to the measured values verifying the functionality of the experimental set up.

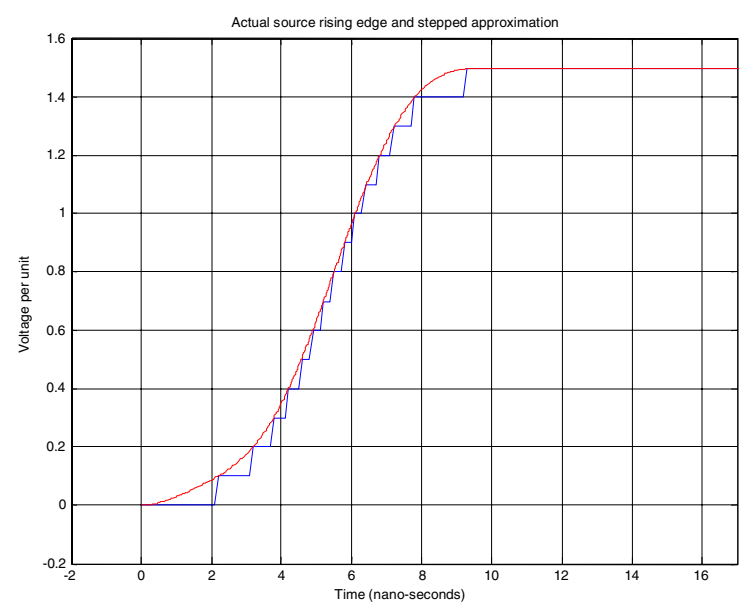

Figure 20. Stepped approximation of source rising edge.

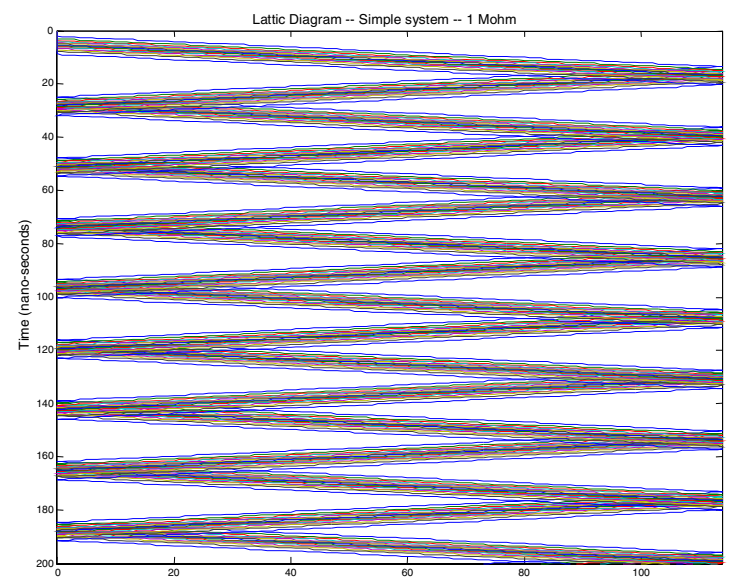

Figure 21. Lattice Diagram of simple system.

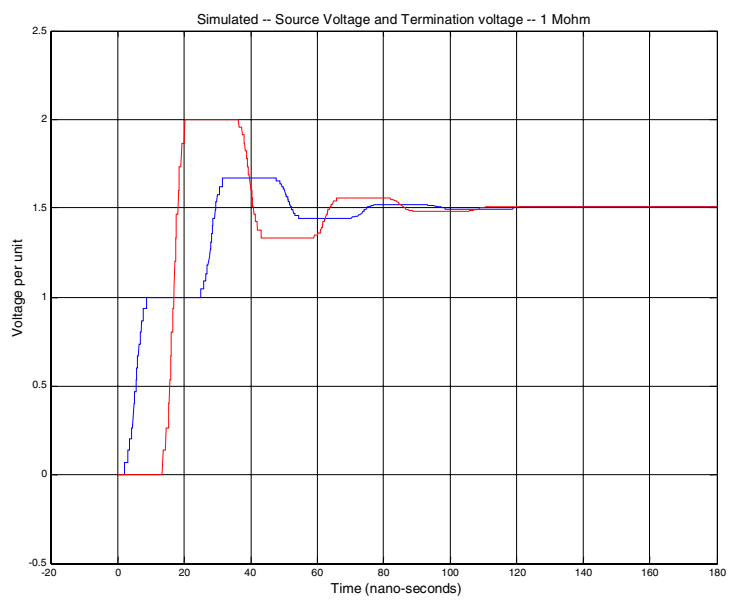

Figure 22. Simulation results at points 'A' and 'B'. 


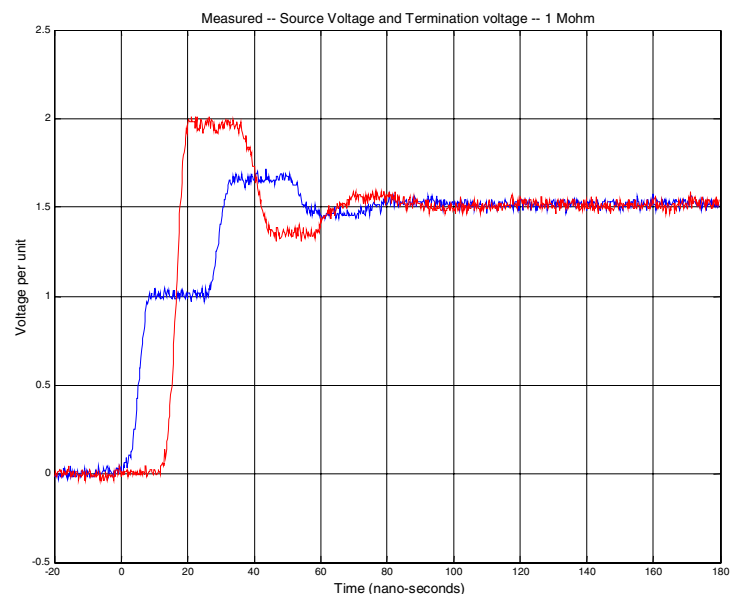

Figure 23. Measured results at points 'A' and 'B'.

\section{MULTIPLE SECTION LATTICE DIAGRAMS}

With the coil parameters obtained, the impedance values associated with the coil sections were determined. These values were then used to determine the reflection and refraction coefficients associated with the boundaries between regions of differing impedance, i.e. slot to overhang. With reflection and refraction coefficients Bewely lattice theory[13] is used to map the propagation of a pulse/surge through the turns of a coil.

The machine under test has four turns per coil, with two conductors per turn, a total of 8 conductors per coil. Here the two parallel conductors are modeled into one conductor having the appropriate impedance value. Initially only one coil is examined under surge conditions. With no inter coil connections both overhang sections are modeled having the same impedance values, with the exception of the connection to the source cable which is handled separately. One complete turn includes four regions of impedance mismatch, slot 'A' - overhang ' $A$ ' - Slot ' $B$ ' - overhang ' $B$ '. Hence, the lattice diagram must have four regions, each representing an impedance region of the coil. As each subsequent turn passes through the same four regions in sequence the lattice is further expanded to have 16 distinct regions. A seventeenth section representing the lead in, half overhang section, at the beginning of the coil is added, which has the same impedance value as a full overhang section. These 17 sections represent the complete coil. As the coil must be connected to a source via a cable, an eighteenth region is added to the front of the lattice. Figure 24 shows the multiple lattice diagram to track pulse propagation through the coil as each discontinuity is reached.

A program using MATLAB was written to evaluate the pulse, the boundary conditions, reflections, refractions, traveling waves and voltages occurring at each location of impedance mismatch. Figure 24 is the lattice result for a test simulation on a simplified coil model, in which the effects of mutual inductive and capacitive coupling were not included. Figure 25 shows the tracking of voltage versus time occurring at the first boundary. Also shown in figure 25 is the source voltage as reference. The voltage at all eighteen boundaries are determined and shown in figure 26 , while only the first and last boundary results are shown in figure 27.

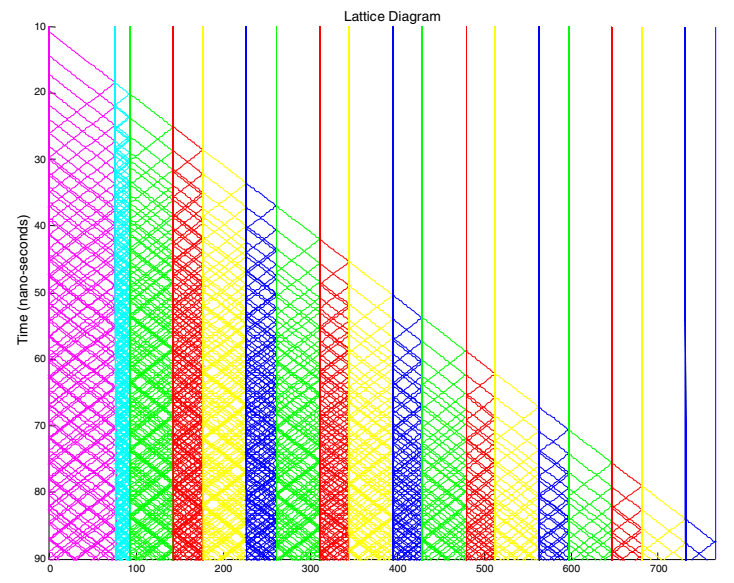

Figure 24. Lattice diagram of 4 turn coil differentiating slot sections from overhang sections, with a supply cable section first.

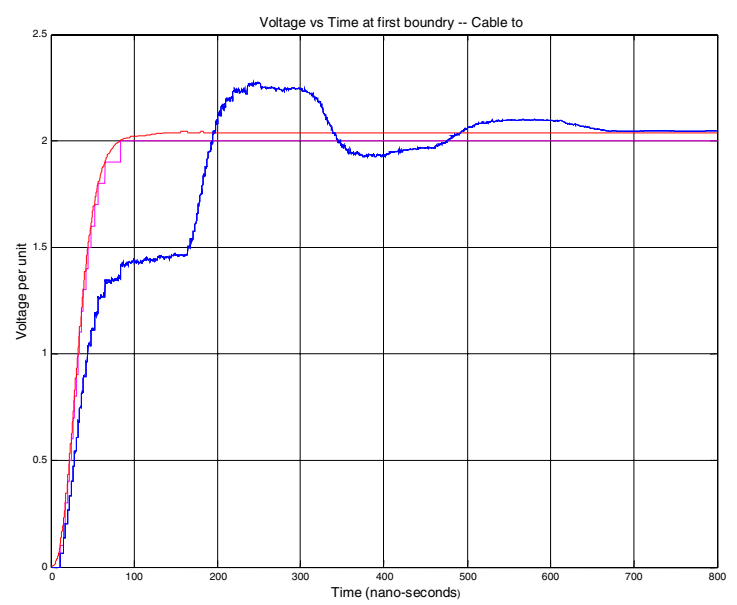

Figure 25. Voltage vs. Time diagram for the first boundary condition.

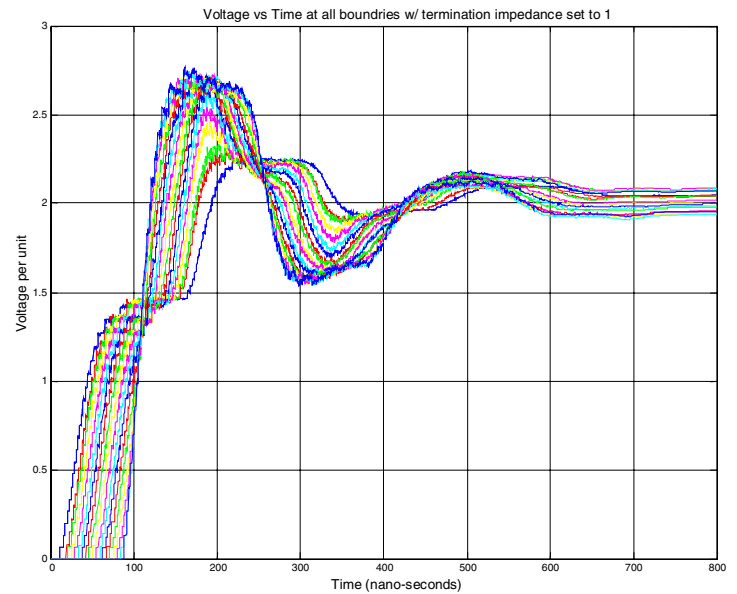

Figure 26. Voltage vs. Time diagram for all 18 boundaries. 


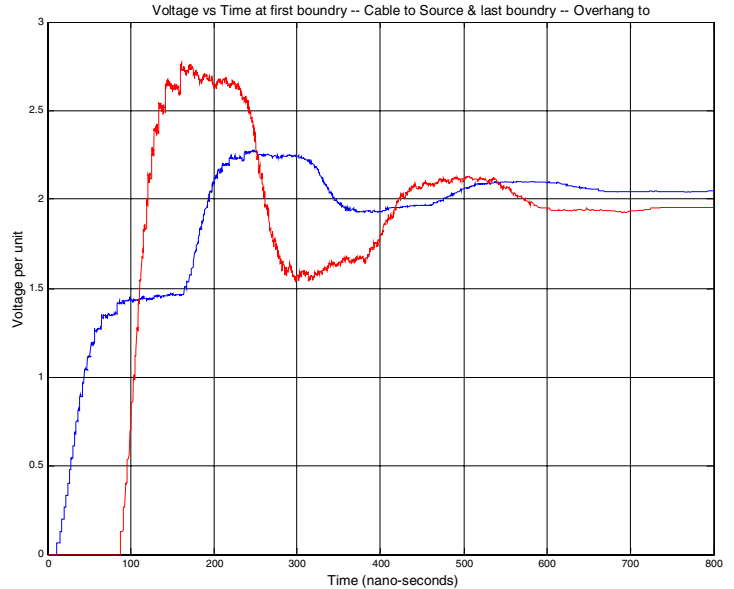

Figure 27. First and last boundary voltages.

\section{PWM PULSE TRACKING}

With single pulse tracking completed, the MATLAB program will be modified to accept variations in the source. A PWM source is added to supply a train of pulses to the coil. The lattice diagram tracks multiple pulses traveling in time at the impedance boundaries. With several pulses entering the coil and being reflected and refracted along with the variations in velocity of propagation from one turn section to another, there is a much greater chance that several reflections will arrive at a boundary simultaneously causing greater overvoltages than predicted in earlier works.

Further use of the PWM lattice program allows the variation in PWM strategies to be tested, identifying which are most benign to the coil insulation.

\section{CONCLUSIONS}

This work makes contributions on the measurement of motor parameters for surge propagation studies.

A single coil of the machine is divided into regions of differing impedance namely the slot and the overhang regions. Detailed parameter measurements are made of each turn within each region, to include turn to ground and turn to turn capacitance and self and mutual inductances. This is then used to determine the surge impedance of each section.

Bewely lattice theory is extended to track surge propagation within the coil. Boundary voltages located at the impedance mismatch are calculated to determine the magnitude of the overvoltages occurring within each turn of a coil.

Future work will focus on PWM interaction inside the windings

\section{REFERENCES}

[1] Tianting Ren, "Analysis of Voltage Source Inverters Operating With Long Cables", M.S. Thesis, Clarkson University, 1997
[2] R. J. Kerkman, D. Leggate and G. L. Skibinski, "Interaction of Drive Modulation and Cable Parameters on AC Motor Transients", IEEE Transactions on Industry Applications, Vol. 33, No. 3, May/June 1997, pp 722-731

[3] A. Von Jouanne, D. A. Rendusare, P. N. Enjeti and J. W. Gray, "Filtering Techniques to Minimize the Effect of Long Motor Leads on PWM Inverter-Fed AC Motor Drive Systems", IEEE Transactions on Industry Applications, Vol. 32, No. 4, July/August 1996, pp 919926

[4] A. H. Bonnett, "Analysis of the Impact of Pulse-Width Modulated Inverter Voltage Waveforms on $\mathrm{AC}$ Induction Motors", IEEE Transactions on Industry Applications, Vol. 32, No. 2, March/April 1996, pp 386392

[5] P. G. McLaren and M. H. Abdel-Rahman, "Modeling of Large AC Motor Coils for Steep-Fronted Surge Studies", IEEE Transactions on Industry Applications, Vol. 24, No. 3, May/June 1988, pp. 422-426

[6] M. T. Wright, S. J. Yang and K. McLeay. "General theory of fast-fronted interturn voltage distribution in electrical machine windings", IEE Proceedings, Vol. 130, Pt. B, No. 4, July 1983, pp. 245-256

[7] L. M. Wedepohl, "Application of matrix methods to the solution of traveling wave phenomena in polyphase systems", IEEE Proceedings, Vol. 110, No. 12, December 1963, pp. 2200-2212.

[8] P. G. McLaren and H. Oraee, "Surge Voltage Distribution in Line-End Coils of Induction Machines", IEEE Transactions on Power Apparatus and Systems, Vol. PAS-104, No. 7, July 1985, pp. 1843-1848

[9] K. J. Cornick and T. R. Thompson, "Steep-Fronted Switching Voltage Transients and Their Distribution in Motor Windings Part 2: Distribution of Steep-Fronted Switching Voltage Transients in Motor Windings", IEE Proceedings, Vol. 129, pt. B, No. 2, March 1982, pp. 5663

[10]K. J. Cornick and T. R. Thompson, "Steep-Fronted Switching Voltage Transients and Their Distribution in Motor Windings Part 1: System Measurements of SteepFronted Switching Voltage Transients", IEE Proceedings, Vol. 129, pt. B, No. 2, March 1982, pp. 4555

[11]W. W. L. Keerthipala and P. G. McLaren, "A Multiconductor Transmission Line Model for Surge Propagation Studies in Large A.C. Machine Windings", Midwest Symposium on Circuits and Systems, V2, August 12-15, 1990, pp.629-632

[12] W. W. L. Keerthipala and P. G. McLaren, "The Effects of Laminations on Steep Fronted Surge Propagation in Large A.C. Motor Coils", IEEE Transactions on Energy Conversion, Vol. 5, No. 1, March 1990, pp. 84-90

[13] L. V. Bewely, "Traveling Waves On Transmission Systems", Dover Edition, Dover Publications, Inc. New York, 1963 